\title{
Uso de etamsilato para reducir el sangrado posoperatorio y el índice de transfusión en la artroplastia total de cadera. Ensayo clínico controlado
}

\author{
Use of ethamsylate to reduce postoperative bleeding and transfusion index in total hip \\ arthroplasty. A randomized clinical trial
}

\author{
Tomás A. Ramos-Sánchez, Tomás Ramos-Morales, Rodolfo Morales-Avalos*, Jaime Blázquez-Saldaña, \\ Víctor M. Peña-Martínez y Félix Vílchez-Cavazos \\ Módulo de Cadera, Miembro Inferior y Remplazo Articular, Servicio de Ortopedia y Traumatología, Hospital Universitario Dr. José Eleuterio \\ González, Universidad Autónoma de Nuevo León, Monterrey, N.L. México
}

\begin{abstract}
Resumen
Antecedentes: El sangrado secundario es una de las principales causas de morbilidad después de la cirugía. El etamsilato se ha utilizado con buenos resultados para disminuir el sangrado en diversas patologías, como metrorragias, sangrado intraventricular, prostatectomías, cirugías de catarata y amigdalectomías. El objetivo de este estudio fue evaluar la efectividad del etamsilato para disminuir el sangrado en la cirugía de reemplazo total de cadera. Método: La población se dividió en dos grupos. En el grupo control se realizó la hemostasia de manera convencional; en el grupo experimental se administró etamsilato. Resultados: Se incluyeron 34 pacientes, de los cuales 17 fueron aleatorizados al grupo de etamsilato y 17 al grupo control. No hubo diferencias en las características de la población entre los dos grupos. Al comparar los valores de hemoglobina preoperatoria y a las 24, 48 y 72 horas posquirúrgicas entre ambos grupos, no se encontraron diferencias estadísticamente significativas. Tampoco hubo diferencia en el hematocrito ni en la cuantificación del gasto por drenaje a las 24 y 48 horas. Hubo tres pacientes transfundidos en el grupo de etamsilato y siete en el grupo de control, lo cual no difirió significativamente $(p=0.62)$. Conclusión: En este estudio no se demostró un efecto sobre la reducción de la hemorragia en pacientes sometidos a reemplazo total de cadera con el uso de etamsilato.
\end{abstract}

PALABRAS CLAVE: Artroplastia total de cadera. Sangrado posoperatorio. Etamsilato. Transfusión.

\begin{abstract}
Background: Secondary bleeding is one of the leading causes of morbidity after the surgery. Ethamsylate has been used with good results to decrease bleeding in various pathologies such as metrorrhagia, intraventricular bleeding, prostatectomies, cataract surgeries and tonsillectomies. The objective of this study was to evaluate the effectiveness of the hemostatic agent ethamsylate to decrease bleeding in total hip replacement surgery. Method: The population were divided into two groups, in the control group was performed the hemostasis conventionally; in the experimental group ethamsylate was administered. Results: A total of 34 patients were included, of whom 17 were randomized to the group of ethamsylate and 17 randomized to the control group. There were no differences in the characteristics of the population between the two groups. Comparing preoperative hemoglobin levels and at 24, 48 and 72 postsurgical hours between the control group and ethamsylate group
\end{abstract}

\footnotetext{
Correspondencia:

*Rodolfo Morales-Avalos

Madrid, 113

Col. Riberas de San Nicolás

Fecha de recepción: 23-01-2018

Cir Cir. 2018;86:270-276

C.P. 66430 , San Nicolás de los Garza, N.L., México

Fecha de aceptación: 24-04-2018

E-mail: rodolfot59@ hotmail.com

DOI://dx.doi.org/10.24875/CIRU.M18000043

Contents available at PubMed

www.cirugiaycirujanos.com
} 
there was no statistically significant difference. There was also no difference in the levels of hematocrit. In the quantification of expenditure by the drainage there was no difference between the groups at 24 and 48 hours. There were three patients transfused in the ethamsylate group and seven in the control group, which did not differ significantly $(p=0.62)$. Conclusion: $A n$ effect on the reduction of bleeding in patients undergoing total hip replacement with the use of hemostatic agent ethamsylate was not demonstrated in this study.

KEY WORDS: Postoperative bleeding. Total hip arthroplasty. Ethamsylate. Transfusion.

\section{Introducción}

El reemplazo total de cadera, también conocido como artroplastia total de cadera (ATC), es una cirugía ortopédica que tiene como objetivo reemplazar la articulación de la cadera por un implante ortopédico. La indicación principal para una artroplastia de cadera sigue siendo la artrosis en etapa avanzada, cuando el tratamiento conservador ha fracasado en aliviar el dolor o este limita las actividades de la vida diaria'. La necrosis avascular con colapso segmentario de la cabeza del fémur y las fracturas desplazadas del cuello del fémur en pacientes mayores de 60 años se han convertido en otras indicaciones frecuentes. Se ha demostrado que la ATC otorga un mejor resultado funcional y menos complicaciones en comparación con las técnicas tradicionales de fijación interna o hemiartroplastia para fracturas desplazadas'. Existen diferentes prótesis de cadera constituidas por un componente acetabular y un componente femoral. La ATC es uno de los procedimientos electivos que se realizan con mayor frecuencia en el mundo. La prevalencia se ha incrementado drásticamente en los últimos años debido a un aumento en la esperanza de vida de la población².

Después de una ATC, los extremos óseos que fueron seccionados y quedan expuestos al no ser cubiertos por los implantes son un factor contribuyente muy importante del sangrado posoperatorio ${ }^{3}$. El control del sangrado a través del hueso expuesto no puede realizarse con electrocauterio ni por ligadura de vasos ${ }^{4}$. Además, el uso de agentes antiplaquetarios y anticoagulantes para reducir los incidentes tromboembólicos incrementan las complicaciones por sangrado. La pérdida de sangre perioperatoria asociada a la ATC es una de las principales causas de morbilidad posoperatoria, incluyendo dolor, disminución en el rango de movilidad e incremento en los requerimientos de transfusiones alogénicas, lo cual puede resultar en incrementos sustanciales de costos y complicaciones significativas, como infección posoperatoria, retraso en la recuperación del paciente, estancias hospitalarias prolongadas e incremento de la mortalidad 5 . Se ha reportado que la pérdida de sangre perioperatoria en cirugía de reemplazo total de cadera primario puede encontrarse en el rango de 1 a 2 litros $^{6}$, lo cual equivale a $4 \pm 1.5 \mathrm{~g} / \mathrm{dl}$ de hemoglobina. Debido a ello, la anemia posoperatoria en pacientes sometidos a una ATC puede llegar a presentarse hasta en el $87 \pm$ $10 \%$. La alta incidencia reportada de anemia posoperatoria conlleva una mayor tasa de transfusión en estos pacientes, la cual se reporta puede llegar hasta un $26 \%$, dependiendo de la experiencia del centro donde se realice este procedimiento. En los EE.UU. se administran anualmente 24 millones de componentes sanguíneos ${ }^{3,8}$. En el área de la ortopedia se usan el $10 \%$ de todos los paquetes globulares transfundidos, de los cuales el $39 \%$ se usan en artroplastias de cadera y de rodilla ${ }^{9}$. En Inglaterra, las artroplastias de cadera y de rodilla consumen el $8 \%$ de todas las unidades sanguíneas transfundidas ${ }^{10}$. Las transfusiones alogénicas han sido el método estándar para manejar la anemia por pérdida aguda de sangre secundaria a ATC. Sin embargo, y a pesar de protocolos muy estrictos de manejo, las transfusiones alogénicas conllevan riesgos inherentes significativos, que incluyen transmisión viral, incompatibilidad del grupo ABO y hemólisis, entre otros ${ }^{11,12}$.

Aunque existe un gran número de estrategias para el manejo del sangrado perioperatorio, hay poca literatura disponible que avale su uso. Debido a las potenciales complicaciones y eventos adversos que se asocian con la pérdida de sangre y las transfusiones de sangre alogénica ${ }^{2}$, es imperativo explorar nuevas estrategias de conservación y manejo para reducir el sangrado perioperatorio.

El etamsilato se usa como agente hemostático desde hace más de 40 años en sangrado intraventricular en pacientes prematuros y en hemorragia uterina anormal. Se ha utilizado también para disminuir el sangrado transoperatorio en adenoidectomías, prostatectomías, cirugías de catarata y cirugías maxilofaciales y dentales ${ }^{13}$. En los últimos estudios acerca de su mecanismo de acción se ha encontrado que promueve la agregación de los leucocitos y las plaquetas cuando la sangre se encuentra en contacto con algún 
segmento vascular dañado, por lo que existe poco riesgo de eventos adversos tromboembólicos ${ }^{14}$. Los mecanismos por los que promueve la agregación de leucocitos y plaquetas hasta el momento conocidos son dos. El primero que fue demostrado es que aumenta la expresión de P-selectina en las membranas plaquetarias, lo que promueve la agregación de las plaquetas $^{15}$. El otro mecanismo es el aumento de la expresión en la membrana de los leucocitos de P-selectina glucoproteína ligando 1 (PSGL-1), lo que promueve la formación de agregados de leucocitos y plaquetas $^{14}$. Existe poca experiencia en el uso del etamsilato en las cirugías ortopédicas, por lo que el objetivo del presente estudio es determinar su eficacia como agente hemostático en las cirugías de ATC comparado con placebo.

\section{Método}

Se realizo un ensayo clínico controlado con un diseño aleatorizado, comparativo, prospectivo y experimental.

\section{Población de estudio}

Utilizando una fórmula para prueba de hipótesis y diferencia de dos medias, con un valor z $\alpha$ de 1.96 con nivel de significancia del $95 \%$ para dos colas, y un valor $z \beta$ de 0.84 con una potencia del $80 \%$, se obtuvo una muestra de 17 participantes por grupo. Los criterios de inclusión fueron personas mayores de 30 años, sometidas a un reemplazo total de cadera primario debido a osteoartrosis, necrosis avascular de cadera o fractura transcervical de cadera. A todos los participantes se les hizo firmar un consentimiento informado en el que expresaban su deseo de participar en el estudio. Los criterios de exclusión se aplicaron a participantes con discrasias sanguíneas, déficit cognitivo, alérgicos al etamsilato o con eventos trombóticos en los últimos 6 meses.

\section{Selección y aleatorización de los pacientes}

La aleatorización de los pacientes para el grupo de tratamiento y el grupo control se realizó mediante un sistema de tabla en la página de internet www.randomization.com. A los pacientes que se asignaron al grupo experimental se les administró etamsilato, y a los que se incluyeron en el grupo control no se les administró ningún medicamento.

\section{Realización del reemplazo total de cadera y aplicación del medicamento}

Las cirugías se realizaron con el paciente en decúbito lateral y bajo anestesia tipo bloqueo epidural y sedación. Se realizó sutura por planos, realizando la reinserción de la porción anterior del músculo glúteo medio. Se realizó el cierre de la fascia lata y se colocó un drenaje tipo Drenovac ${ }^{\circledR}$ de 1/4 de pulgada por encima de esta última y por debajo del tejido celular subcutáneo, el cual se dejó a succión posterior a 4 horas de pinzamiento. Se realizó la sutura del tejido celular subcutáneo de la manera acostumbrada y se cerró la piel con grapas. Al final se colocó un parche con gasa e Hypafix ${ }^{\circledR}$. En todos los pacientes se utilizaron medidas tradicionales para evitar el sangrado, tales como el uso de electrocauterio (Valleylab Force EZ) y la aplicación de presión directa sobre los puntos sangrantes.

Se conservó el medicamento a temperatura ambiente, a no más de $25^{\circ} \mathrm{C}$, y protegido de la luz. Se aplicaron $500 \mathrm{mg}$ (dos ampolletas de $250 \mathrm{mg}$ sin diluir, administradas en forma lenta) intravenosos de etamsilato (Dicynone ${ }^{\circledR}$ ) en los 30 minutos previos al procedimiento quirúrgico y $500 \mathrm{mg}$ al momento de realizar la osteotomía del cuello femoral. Todos los pacientes fueron manejados con heparina de bajo peso molecular por vía subcutánea en los primeros 2 días posoperatorios, y posteriormente fueron cambiados a un inhibidor del factor $\mathrm{X}$ activado por vía oral por otros 30 días como profilaxis para tromboembolia venosa. Todos los pacientes iniciaron terapia física con ejercicios en las primeras 24 horas y todos se movilizaron dentro de las primeras 48 horas.

\section{Evaluación de los resultados obtenidos: hemoglobina, hematocrito, cuantificación del drenaje y tasa de transfusión}

Se obtuvieron los datos generales del paciente: edad, sexo, peso, talla, índice de masa corporal y antecedentes personales. Se midieron la hemoglobina y el hematocrito preoperatorios y a las $6 \mathrm{~h}$ a.m. de los días 1, 2 y 3 del posoperatorio. Se cuantificó el gasto por el drenaje a las 24 y 48 horas posoperatorias y luego se retiró. Se midió la tasa de transfusión, según el valor de hemoglobina designado. Por último, se evaluó la escala funcional (escala visual análoga) a las 24, 48 y 72 horas (Tabla 1). En el posoperatorio se transfundió a todo paciente con valores de hemoglobina $<9 \mathrm{mg} / \mathrm{dl}$ con síndrome anémico. 
Tabla 1. Variables evaluadas y tiempos en que fueron evaluadas

\begin{tabular}{lllll}
\hline Variable & Preoperatorio & Día 1 posoperatorio & Día 2 posoperatorio & Día 3 posoperatorio \\
\hline Hemoglobina & 1 toma & 1 toma $6 \mathrm{~h} \mathrm{a} . \mathrm{m}$. & 1 toma $6 \mathrm{~h} \mathrm{a} . \mathrm{m}$. & 1 toma $6 \mathrm{~h}$ a.m. \\
Hematocrito & 1 toma & 1 toma $6 \mathrm{~h} \mathrm{a.m.}$ & 1 toma $6 \mathrm{~h} \mathrm{a.m}$. & 1 toma $6 \mathrm{~h}$ a.m. \\
Gasto drenaje & & $24 \mathrm{~h}$ posquirúrgicas & $48 \mathrm{~h}$ posquirúrgicas & \\
Escala visual análoga & 1 medición & 1 medición & 1 medición & 1 medición \\
\hline
\end{tabular}

\section{Resultados}

Se incluyeron 34 pacientes, de los cuales 17 se aleatorizaron al grupo de etamsilato y 17 al grupo control. Se analizaron las características de la población de cada grupo de estudio (edad, sexo y diagnóstico), sin encontrar diferencias entre ambos grupos. La edad media en el grupo control fue de $60.90 \pm$ 13.01 años, y en el grupo de etamsilato fue de 58.57 \pm 13.44 años, sin que hubiera diferencia estadísticamente significativa $(p=0.67)$. En cuanto al sexo, no hubo diferencia estadísticamente significativa entre el grupo control y el grupo etamsilato $(p=0.82)$ (Tabla 2).

Los diagnósticos incluidos fueron fractura de fémur, coxartrosis y necrosis avascular de cadera. En el grupo control, siete $(41.18 \%)$ pacientes tenían fractura transcervical de cadera, siete $(41.18 \%)$ tenían coxartrosis y tres (17.64\%) tenían necrosis avascular de cadera. En el grupo de etamsilato se operaron cinco $(29.41 \%)$ pacientes con fractura transcervical de cadera, siete $(41.18 \%)$ con coxartrosis y cinco $(29.41 \%)$ con necrosis avascular de cadera; no hubo diferencias estadísticamente significativas $(p=0.81)$.

Se realizaron mediciones de los valores de hemoglobina previamente a la cirugía y en los días 1, 2 y 3 del posoperatorio. Se encontró un valor preoperatorio de $13.54 \pm 1.17 \mathrm{~g} / \mathrm{dl}$ en el grupo control y $13.60 \pm$ $1.41 \mathrm{~g} / \mathrm{dl}$ en el grupo de etamsilato $(p=0.9)$. En el día 1 posterior a la cirugía se encontró un valor de hemoglobina de $9.69 \pm 0.95 \mathrm{~g} / \mathrm{dl} \mathrm{y} 10.15 \pm 1.82 \mathrm{~g} / \mathrm{dl}$, para el grupo control y el grupo de etamsilato $(p=0.48)$. En el día 2 del posoperatorio los valores encontrados en el grupo control y el grupo de etamsilato fueron de $9.26 \pm 1.25 \mathrm{~g} / \mathrm{dl}$ y $9.46 \pm 1.53 \mathrm{~g} / \mathrm{dl}$, respectivamente ( $p=0.74$ ). Por ultimo en el día 3 del posoperatorio se encontraron valores de $8.99 \pm 1.42 \mathrm{~g} / \mathrm{dl}$ en el grupo control y $9.01 \pm 1.06 \mathrm{~g} / \mathrm{dl}$ en el grupo de etamsilato $(p=0.97)$. A pesar de que las cifras de hemoglobina fueron menores en el grupo control, esto no tuvo diferencia estadísticamente significativa (Fig. 1).
Tabla 2. Características demográficas de la población incluida en el estudio

\begin{tabular}{lccc}
\hline Características & Control & Etamsilato & $\mathbf{p}$ \\
\hline Edad & $60.90( \pm 13.01)$ & $58.57( \pm 13.44)$ & 0.67 \\
Hombre & $\mathrm{n}=7(41.18 \%)$ & $\mathrm{n}=8(47.06 \%)$ & 0.82 \\
Mujer & $\mathrm{n}=10(58.82 \%)$ & $\mathrm{n}=9(52.94 \%)$ & \\
\hline
\end{tabular}

Se evaluó también el hematocrito de forma previa a la cirugía y en los días 1, 2 y 3 posoperatorios. De forma inicial el hematocrito fue del $41.98 \pm 3.53 \%$ en el grupo control y del $41.42 \pm 4.43 \%$ en el grupo de etamsilato $(p=0.74)$. En el primer día del posoperatorio el hematocrito en el grupo control y en el grupo de etamsilato fue del $29.44 \pm 3.18 \%$ y el $30.76 \pm 5.80 \%$, respectivamente $(p=0.52)$. Al segundo día del posoperatorio, el hematocrito del grupo control fue del 28.0 $\pm 4.12 \%$ y el del grupo de etamsilato fue del $28.49 \pm$ $4.53 \%(p=0.78)$. En el último día, el hematocrito fue del $27.16 \pm 4.54 \%$ en el grupo control y del $26.11 \pm$ $5.91 \%$ en el grupo de etamsilato $(p=0.65)$. A pesar de encontrar una tendencia a un menor hematocrito en el grupo control en los días 1 y 2 del posoperatorio, la diferencia no fue estadísticamente significativa (Fig. 2).

Se cuantificó el gasto del drenaje a las 24 y 48 horas posquirúrgicas (Fig. 3). En la medición realizada a las 24 horas, el gasto fue menor en el grupo control que en el grupo de etamsilato, con $171.33 \pm 166.91 \mathrm{ml}$ y $209.57 \pm 179.14 \mathrm{ml}$, respectivamente, sin que esto fuera estadísticamente significativo $(p=0.61)$. Por el contrario, a las 48 horas se encontró que el gasto del drenaje fue mayor en el grupo control que en el grupo de etamsilato, con $174.44 \pm 137.87 \mathrm{ml}$ y $144.85 \pm$ $135.07 \mathrm{ml}$, respectivamente, sin que esto tuviera significancia estadística $(p=0.61)$

Se midió la tasa de transfusión en el grupo control $y$ en el grupo de etamsilato, encontrando que se transfundieron siete pacientes en el grupo control y tres en el grupo de etamsilato, lo cual no tuvo diferencia estadística $(p=0.62)$. Las indicaciones de las transfusiones fueron en todos los casos unas cifras de hemoglobina $<9 \mathrm{mg} / \mathrm{dl}$ junto con datos de 


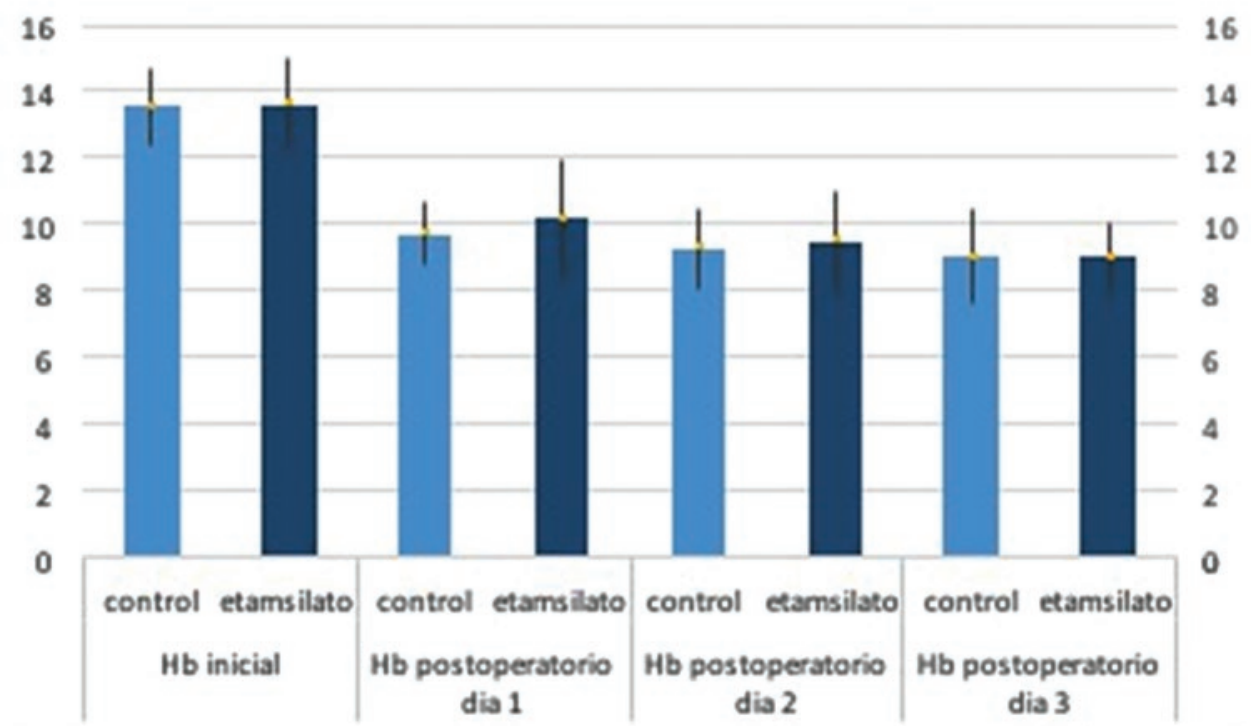

Figura 1. Medias y desviaciones estándar de los valores del hematocrito obtenidos para cada uno de los grupos en los diferentes días de evaluación. Hto: hematocrito.

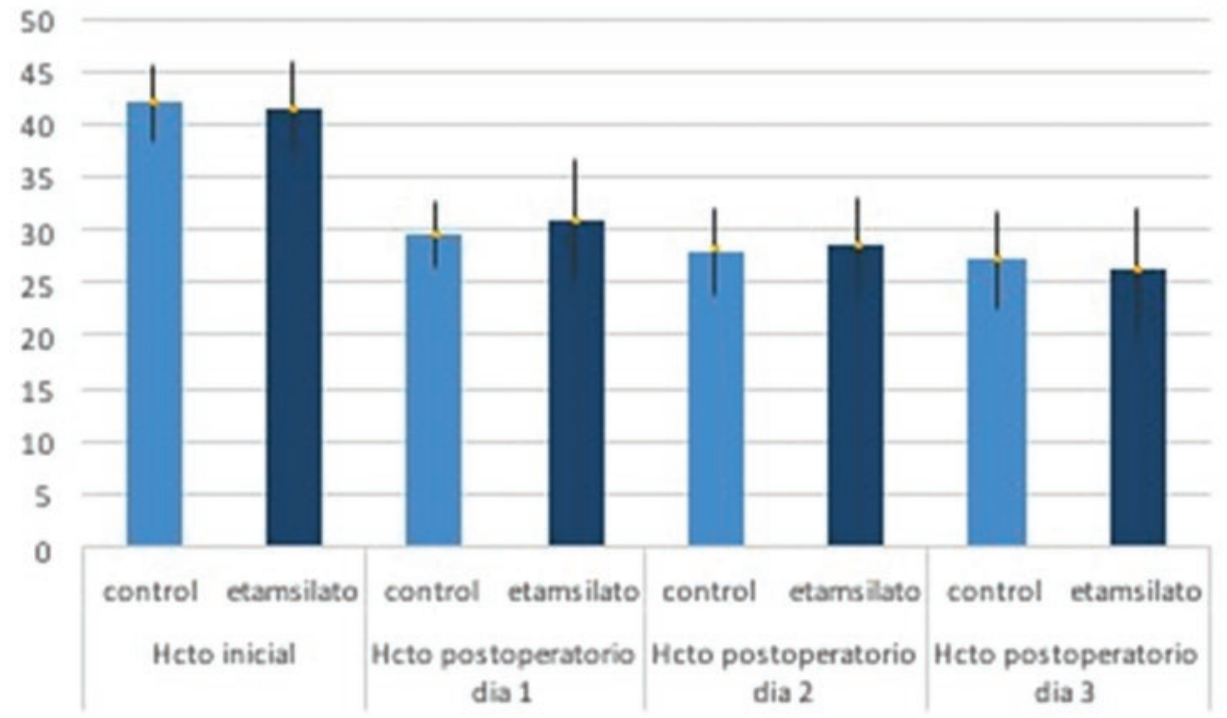

Figura 2. Medias y desviaciones estándar del gasto del drenaje para cada uno de los grupos en los diferentes días de evaluación.

síndrome anémico (mareo, diaforesis, taquicardia, etc.). En nueve de los diez casos se transfundió solo una unidad sanguínea y en un caso fue necesario transfundir dos paquetes globulares.

En el periodo de evaluación, en ninguno de los pacientes incluidos se reportaron complicaciones perioperatorias o posoperatorias (salvo la necesidad de transfusión). No hubo diferencia significativa en los resultados de la escala visual análoga (Tabla 3).
Tabla 3. Escala visual análoga preoperatoria y los días 1,2 y 3 del posoperatorio

\begin{tabular}{lccc}
\hline Escala Visual Análoga & Control & Etamsilato & $\mathbf{p}$ \\
\hline EVA preoperatoria & $6.1( \pm 3.02)$ & $6.3( \pm 2.6)$ & 0.86 \\
EVA día 1 posoperatorio & $3.5( \pm 2.07)$ & $3.6( \pm 2.2)$ & 0.94 \\
EVA día 2 posoperatorio & $2.1( \pm 1.9)$ & $2.7( \pm 1.5)$ & 0.42 \\
EVA día 3 posoperatorio & $1.7( \pm 1.7)$ & $1.5( \pm 2.6)$ & 0.85 \\
\hline EVA: escala visual análoga. & & &
\end{tabular}




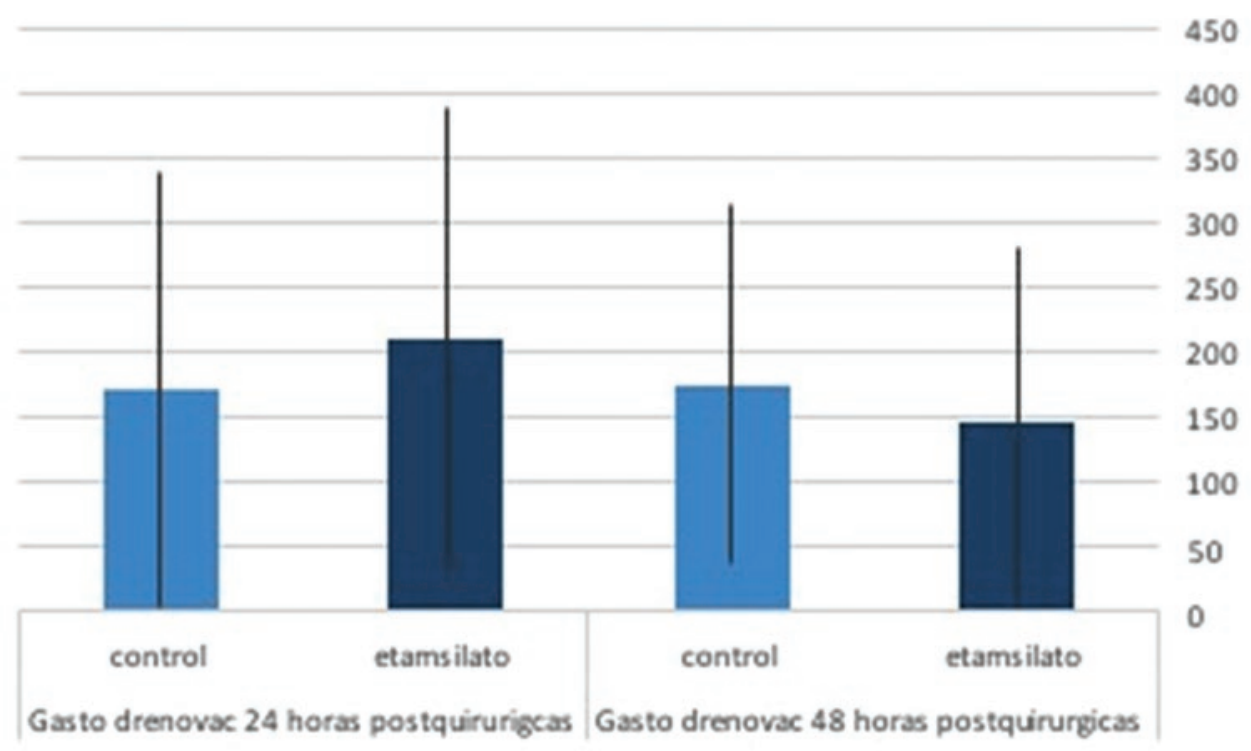

Figura 3. Medias y desviaciones estándar del gasto del drenaje para cada uno de los grupos en los diferentes días de evaluación.

\section{Discusión}

El uso del etamsilato como tratamiento para disminuir el sangrado ha demostrado su eficacia en diversas patologías y procedimientos quirúrgicos no ortopédicos, con un bajo índice de efectos adversos ${ }^{11}$. Sin embargo, no hay mucha evidencia de su eficacia en cirugías ortopédicas. En la literatura solo se encontró un artículo que valora la eficacia del etamsilato en cirugías ortopédicas, en el que se utilizó una dosis similar del medicamento antes de la inducción anestésica ${ }^{16}$. Al igual que en nuestro protocolo, no se encontró que tuviera un efecto positivo en disminuir el sangrado en cirugía de reemplazo total de cadera. En este estudio se propuso como posible explicación de la falta de eficacia que este tipo de procedimiento implica un mayor traumatismo a los tejidos en comparación con otras cirugías en las que se ha demostrado una disminución del sangrado.

No se encontraron diferencias en las características de la población de ambos grupos. Los valores de la hemoglobina y del hematocrito encontrados en nuestro estudio fueron mayores en el grupo de etamsilato que en el grupo control, pero esto no llegó a ser estadísticamente significativo como para demostrar su eficacia. Al comparar los valores de hemoglobina reportados en este artículo con los valores de hemoglobina posquirúrgicos en el grupo control de otros estudios encontramos resultados similares.

Algo que sí pudo haber influido en los resultados de este estudio es que, debido a una disminución no esperada de la cantidad de pacientes candidatos a reemplazo total de cadera en nuestra institución, se incluyeron diversas patologías, como artrosis postraumática, fracturas de cadera y artritis reumatoide, las cuales tienen una tendencia mayor al sangrado y en ocasiones presentan tejido cicatricial y tejido fibroso, lo que se traduce en una mayor manipulación de los tejidos y más tiempo quirúrgico, asociándose a un sangrado mayor, lo cual se reconoce como una limitación del estudio que podría causar sesgos.

Con la realización de este estudio pudo comprobarse el amplio margen de seguridad de este medicamento, ya que no se presentaron eventos adversos en ninguno de los pacientes a los que se administró. El etamsilato es un medicamento que puede seguirse administrando cada 6 horas mientras exista riesgo de sangrado; desconocemos si continuar el medicamento durante este periodo hubiera resultado en alguna diferencia significativa, por lo que podría ser necesaria la realización de más estudios para descartar por completo la eficacia de este medicamento.

A pesar de que no hubo diferencia en la tasa de transfusión entre ambos grupos, la tasa global de transfusión fue del 33.3\%, similar a la descrita en la literatura, por lo cual se debe continuar con la búsqueda de un medicamento seguro, eficaz y accesible para disminuir la tasa de complicaciones asociadas a la ATC.

\section{Conclusiones}

No se encontró diferencia estadísticamente significativa en ninguno de los parámetros a evaluar 
(hemoglobina, hematocrito, transfusiones y cuantificación del gasto del drenaje).

Se requiere la realización de nuevos estudios con mayor número de pacientes, mejor control en los diagnósticos de inclusión y considerando la administración más prolongada del medicamento para descartar por completo su eficacia.

\section{Conflicto de intereses}

Los autores declaran que no tienen ningún conflicto de intereses.

\section{Bibliografía}

1. Bucholz RW. Indicaciones, técnicas y resultados de reemplazo total de cadera en Estados Unidos. Rev Med Clin Condes. 2014;25:760-4.

2. Levine BR, Haughom B, Strong B, Hellman M, Frank RM. Blood management strategies for total knee arthroplasty. J Am Acada Orthop Surg. 2014:22:361-71.

3. Ponnunsamy KE, Thomas TJ, Khnuja HS. Perioperative blood transfusions in orthopaedic surgery. J Bone Joint Surg Am. 2014;96:1836-44.

4. Raut W, Stone MH, Wroblewski BM. Reduction of postoperative blood loss after press-fit condylar knee arthroplasty with use of a femoral intramedullary plug. J Bone Joint Surg Am. 1993;75:1356-7.
5. Sáhn DR. Anemia and patient blood management in hip and knee surgery: a systematic review of the literature. Anesthesiology. 2010;113: 482-95.

6. Bierbaum BE, Callaghan JJ, Galante JO, Rubash HE, Tooms RE, Welch RB. An analysis of blood management in patients having a total hip and knee arthroplasty. J Bone Joint Surg Am. 1999;81:2-10.

7. Spahn DR. Anemia and patient blood management in hip and knee surgery: a systematic review of the literature. Anesthesiology. 2010;113:482-95

8. Goodnough LT, Levy JH, Murphy MF. Concepts of blood transfusion in adults. Lancet. 2013;381:1845-54.

9. Young SW, Marsh DJ, Akhavani MA, Walker CG, Skinner JA. Attitudes to blood transfusion post arthroplasty surgery in the United Kingdom: a national survey. Int Orthop. 2008;32:325-9.

10. Wells AW, Mounter PJ, Chapman CE, Stainsby D, Wallis JP. Where does blood go? Prospective observational study of red cell transfusion in north England. BMJ. 2002;325:803

11. Kirkley SA, Cowles J, Pellegrini VD, Harris CM, Boyd AD, Blumberg N. Blood transfusion and total joint replacement surgery: T helper $2(\mathrm{TH} 2)$ cytokine secretion and clinical outcome. Transfus Med. 1998;8:195-204.

12. Klein HG. How safe is blood, really? Biologicals. 2010;38:100-4.

13. Garay RP, Chiavaroli C, Hannaert P. Therapeutic efficacy and mechanism of action of ethamsylate, a long-standing hemostatic agent. Am J Ther. 2006;13:236-47

14. Hernández MR, Álvarez-Guerra M, Escolar G, Chiavaroli C, Hannaert P Garay RP. The hemostatic agent ethamsylate promotes platelet/leukocyte aggregate formation in a model of vascular injury. Fundam Clin Pharmacol. 2004;18:423-30.

15. Álvarez-Guerra M, Hernández MR, Escolar G, Chiavarolic C, Garaya RP, Hannaer P. The hemostatic agent ethamsylate enhances P-selectin membrane expression in human platelets and cultured endothelial cells. Thromb Res. 2002;107:329-35.

16. Keith MB. Ethamsylate and blood loss in total hip replacement. Anaesthesia. 1979;34:666-70. 